\title{
The stability of a crystal with diamond structure for patchy particles with tetrahedral symmetry
}

\author{
Eva G. Noya, ${ }^{1, a)}$ Carlos Vega $^{2}$ Jonathan P. K. Doye, ${ }^{3}$ and Ard A. Louis ${ }^{4}$ \\ ${ }^{1}$ Instituto de Química Física Rocasolano, Consejo Superior de Investigaciones Científicas (CSIC), Calle \\ Serrano 119, 28026 Madrid, Spain \\ ${ }^{2}$ Departamento de Química Física, Facultad de Ciencias Químicas, Universidad Complutense de Madrid, \\ E-28040 Madrid, Spain \\ ${ }^{3}$ Department of Chemistry, Physical and Theoretical Chemistry Laboratory, University of Oxford, South \\ Parks Road, Oxford OX1 3QZ, United Kingdom \\ ${ }^{4}$ Rudolf Peierls Centre for Theoretical Physics, University of Oxford, 1 Keble Road, Oxford OX1 3NP, \\ United Kingdom
}

(Received 26 March 2010; accepted 26 May 2010; published online 21 June 2010)

\begin{abstract}
The phase diagram of model anisotropic particles with four attractive patches in a tetrahedral arrangement has been computed at two different values of the range of the potential, with the aim of investigating the conditions under which a diamond crystal can be formed. We find that the diamond phase is never stable for our longer-ranged potential. At low temperatures and pressures, the fluid freezes into a body-centered-cubic solid that can be viewed as two interpenetrating diamond lattices with a weak interaction between the two sublattices. Upon compression, an orientationally ordered face-centered-cubic crystal becomes more stable than the body-centered-cubic crystal, and at higher temperatures, a plastic face-centered-cubic phase is stabilized by the increased entropy due to orientational disorder. A similar phase diagram is found for the shorter-ranged potential, but at low temperatures and pressures, we also find a region over which the diamond phase is thermodynamically favored over the body-centered-cubic phase. The higher vibrational entropy of the diamond structure with respect to the body-centered-cubic solid explains why it is stable even though the enthalpy of the latter phase is lower. Some preliminary studies on the growth of the diamond structure starting from a crystal seed were performed. Even though the diamond phase is never thermodynamically stable for the longer-ranged model, direct coexistence simulations of the interface between the fluid and the body-centered-cubic crystal and between the fluid and the diamond crystal show that at sufficiently low pressures, it is quite probable that in both cases the solid grows into a diamond crystal, albeit involving some defects. These results highlight the importance of kinetic effects in the formation of diamond crystals in systems of patchy particles. (C) 2010 American Institute of Physics. [doi:10.1063/1.3454907]
\end{abstract}

\section{INTRODUCTION}

The study of the behavior of anisotropic particles has attracted significant attention in recent years. Initially the interest arose because they were seen as very simplified models of proteins. ${ }^{1-6}$ Even though important advances have been made using simple isotropic models, ${ }^{7,8}$ interactions between proteins are highly anisotropic, ${ }^{9}$ and an improved description of the behavior of proteins can potentially be obtained using models that explicitly incorporate this anisotropy. ${ }^{5,10-15}$ For example, anisotropic models can lead to the stabilization of low density crystals, ${ }^{5,10}$ with packing fractions similar to those typically formed by proteins. ${ }^{9}$ They are also able to reproduce quantitatively the metastable fluidfluid phase separation of globular proteins, ${ }^{11,12}$ whereas isotropic models could only reproduce it qualitatively. In addition, it has been suggested that the kinetics of protein crystallization could be sensitive to the degree of anisotropy. ${ }^{16}$

In the past few years, a number of experimental groups

${ }^{a)}$ Electronic mail: eva.noya@iqfr.csic.es. have developed new methods to produce nanoparticles and colloids with anisotropic shapes or interactions, ${ }^{17-31}$ and this has led to increased interest in anisotropic particles. ${ }^{32-34}$ These experimental developments have motivated many theoretical and simulation studies on how these patchy particles would assemble into crystalline structures ${ }^{5,10,35-38}$ or into clusters with a particular geometry. ${ }^{39-42}$ Much of the work in this latter topic has been also aimed at getting a better understanding of the assembly of virus capsids. ${ }^{39,42}$ One interesting example of the degree of complex behavior that anisotropic particles can exhibit is provided by one patch particles that mimic Janus particles (colloidal particles whose surface is divided into two areas with different chemical

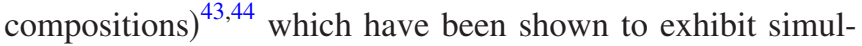
taneously gas-liquid phase separation and the formation of micelles. ${ }^{44}$ This recent example illustrates the potential richness of the behavior that anisotropic models can exhibit, and that there is much still to be learnt.

In previous work, we have studied the crystallization behavior of patchy particles in two and three dimensions and found that the geometry of the patches strongly affects 
crystallization. ${ }^{10}$ For example, crystallization can be frustrated when the patches are not straightforwardly compatible with a crystalline structure, e.g., five regularly arranged patches for two dimensional particles. However, perhaps more surprising is that even in cases where the symmetry of the particles is compatible with a crystalline structure, there can be strong variations in the crystallization behavior. In particular, we found that whereas a simple-cubic structure can be easily obtained by quenching a fluid of six-patch octahedral particles, it is difficult to obtain a diamond structure by quenching a fluid of four-patch tetrahedral particles. ${ }^{10}$ Similarly, Zhang et al. ${ }^{37}$ were only able to obtain a diamond crystal from such tetrahedral patchy particles when a crystal seed was inserted in the simulation box or when the model potential included torsional interactions. By studying the geometry of the clusters formed by the octahedral and tetrahedral model particles, Doye et al. ${ }^{10}$ attributed the different behaviors of the two systems to the frustration between the local order in the fluid and the global crystalline order for the tetrahedral particles. Given the possible applications of a diamond colloidal crystal in photonics due to its predicted optical band gap, ${ }^{45-47}$ and the growing interest in patchy particles, in general, it would be interesting to study in more detail the crystallization behavior of the tetrahedral patchy particles.

In this work, the phase diagram of model tetrahedral particles is investigated by means of computer simulation. Even though there is a very recent study on the phase behavior of tetrahedral patchy particles, ${ }^{36}$ the present work differs in the model used to describe patchy particles. Romano et $a l .{ }^{36}$ used the Kern-Frenkel (KF) model, ${ }^{4}$ in which particles are described as hard spheres with some attractive sites modeled as square wells, whereas in this work particles are modeled using a generalized Lennard-Jones (LJ) potential modulated by Gaussian functions at the location of the patches. ${ }^{10}$ This model potential (and modified versions of it) has been previously used to study crystallization, ${ }^{10}$ phase behavior, ${ }^{35}$ and the self-assembly of clusters of patchy particles with various symmetries. ${ }^{40-42}$ Comparisons between the present work and that of Romano et al. will allow us to discern the intrinsic behavior of tetrahedral particles from particular behavior that arises from the specific shape of the model potential.

\section{METHOD}

\section{A. Model}

Anisotropic particles are modeled using a pair potential that consists of the generalized LJ repulsive core and an attractive tail modulated by an angular function that depends on how directly the patches point at each other. The interaction between two particles $i$ and $j$ depends on the distance vector between them $\left(\mathbf{r}_{i j}\right)$ and on their orientation $\left(\boldsymbol{\Omega}_{i}\right.$ and $\left.\boldsymbol{\Omega}_{j}\right)$,

$$
V\left(\mathbf{r}_{i j}, \boldsymbol{\Omega}_{i}, \boldsymbol{\Omega}_{j}\right)=\left\{\begin{array}{cc}
V_{\mathrm{LJ}}\left(r_{i j}\right), & r_{i j}<\sigma_{\mathrm{LJ}} \\
V_{\mathrm{LJ}}\left(r_{i j}\right) V_{\text {ang }}\left(\hat{\mathbf{r}}_{i j}, \boldsymbol{\Omega}_{i}, \boldsymbol{\Omega}_{j}\right), & r_{i j} \geq \sigma_{\mathrm{LJ}},
\end{array}\right.
$$

where $V_{\mathrm{LJ}}\left(r_{i j}\right)$ is a generalized $2 n-n \mathrm{LJ}$ potential,

$$
V_{\mathrm{LJ}}\left(r_{i j}\right)=4 \epsilon\left[\left(\frac{\sigma_{\mathrm{LJ}}}{r_{i j}}\right)^{2 n}-\left(\frac{\sigma_{\mathrm{LJ}}}{r_{i j}}\right)^{n}\right],
$$

and $\sigma_{\mathrm{LJ}}$ is the distance at which the LJ potential passes through zero. Our purpose is to study the phase behavior for the usual 12-6 LJ model. However, we are also interested in investigating the effects of the range of the potential, which can be tuned by modifying the exponents of the generalized LJ potential. Although the depth of the potential is independent of the value of $n$, the position of the potential minimum varies and is at $2^{1 / n} \sigma_{\mathrm{LJ}}$. The phase behavior of the 20-10 model will also be investigated in this work. For this model, the position of the minimum is $1.0718 \sigma_{\mathrm{LJ}}$, whereas for the usual $12-6 \mathrm{LJ}$ potential it is $1.1225 \sigma_{\mathrm{LJ}}$.

The generalized LJ potential is modulated by the factor $V_{\text {ang }}\left(\hat{\mathbf{r}}_{i j}, \boldsymbol{\Omega}_{i}, \boldsymbol{\Omega}_{j}\right)$, which is a product of Gaussian functions that depend on the alignment of the patches with the interparticle vector,

$$
V_{\text {ang }}\left(\hat{\mathbf{r}}_{i j}, \boldsymbol{\Omega}_{i}, \boldsymbol{\Omega}_{j}\right)=\exp \left(-\frac{\theta_{i j k_{\min }}^{2}}{2 \sigma_{\mathrm{pw}}^{2}}\right) \exp \left(-\frac{\theta_{j i l_{\text {min }}^{2}}}{2 \sigma_{\mathrm{pw}}^{2}}\right),
$$

where $\theta_{i j k}$ is the angle between $\hat{\mathbf{r}}_{i j}$ and patch $k$ on particle $i$ and $k_{\min }$ is the patch that minimizes this angle. Thus, $V_{\text {ang }}$ $=1$ when two patches directly point at each other. The parameter $\sigma_{\mathrm{pw}}$ is a measure of the width of the patches, with $2 \sqrt{2} \sigma_{\mathrm{pw}}$ being the full width at half maximum of the Gaussian. For computational efficiency, this potential was truncated and shifted at a cutoff distance of $2.5 \sigma_{\mathrm{LJ}}$.

In this work, we study particles with four tetrahedrally arranged patches with a patch width of $\sigma_{\mathrm{pw}}=0.3 \mathrm{rad}$. In a previous study of octahedral particles with six patches using the 12-6 model it was found that this patch width is sufficiently narrow to stabilize a low density simple-cubic crystal. ${ }^{35}$ Therefore, we expect these particles to represent promising candidates for the formation of a diamond crystal at low pressure. Throughout this paper, all quantities will be given in reduced units, i.e., $u^{*}=u / \epsilon, T^{*}=k_{B} T / \epsilon, \rho^{*}=\rho \sigma_{\mathrm{LJ}}^{3}$, and $p^{*}=p \sigma_{\mathrm{LJ}}^{3} / \epsilon$.

\section{B. Solid structures}

As mentioned before, the tetrahedral geometry of the particles was chosen in order to explore the possibility that at sufficiently low temperatures and pressures, the formation of a diamond crystal would be favored. In the diamond lattice each of the four patches point directly at one of the four nearest neighbors [Fig. 1(a)]. As the four patches are able to form a "perfect" bond (i.e., with energy $-\epsilon$ ), the energy will be minimized in this structure.

However, the diamond solid has a very low density and it is expected that a body-centered-cubic (bcc) structure [Fig. 1(b)] will be competitive with diamond. Specifically, in the diamond crystal there is enough free space to interpenetrate a second diamond lattice displaced with respect to the first one by a vector $(a / 2, a / 2, a / 2)$, with $a$ being the unit cell parameter of the cubic diamond lattice, and the density of the resulting bec crystal is exactly twice that of the diamond structure. When the distance between nearest neighbors is equal to $\sigma_{\mathrm{LJ}}$, i.e., when the repulsive cores of the particles touch, 
(a)

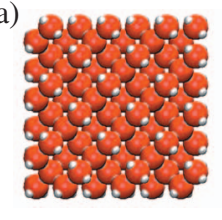

(b)

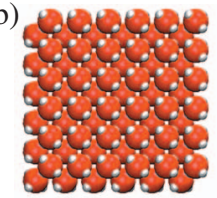

(c)

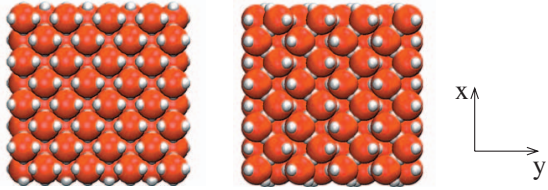

FIG. 1. Orientationally ordered crystal structures for tetrahedral patchy particles: (a) diamond, (b) bcc, and (c) fcc-o. Two views are shown in each case, with the picture on the right corresponding to a rotation of the structure by [(a) and (b)] $\pi / 4$ and (c) $\pi / 2$ about the $x$ axis.

then the densities of the diamond and bcc crystals are $\rho^{*}$ $=0.6495$ and 1.2990, respectively. However, the energetically preferred nearest-neighbor distance corresponds to the minimum in the potential, and the corresponding density of the crystals are $\rho^{*}=0.4593$ and 0.9186 for the $12-6$ model and $\rho^{*}=0.5276$ and 1.0551 for the 20-10 model. Consequently, for both the models that we consider, it is always possible to interpenetrate the two sublattices without any deformation of the two lattices, i.e., with no energy cost, although there is slightly less room for the sublattices to vibrate in the shorter-ranged model. This situation is somewhat similar to that found for the octahedral particles, in which case the bcc structure is formed by interpenetrating two simple-cubic lattices. ${ }^{35}$ However, for the octahedral particles, the two simple-cubic lattices have to be expanded slightly with respect to their ideal densities in order to interpenetrate without the repulsive cores of the particles overlapping, and so there is an associated energy penalty. ${ }^{35}$

At zero temperature and zero pressure, the most stable solid will be that with least potential energy. For the patch width studied in this work, there is a small attractive interaction between the two diamond sublattices in the bcc structure which slightly lowers the energy of the bcc solid with respect to that of the diamond. Therefore, at zero temperature and at zero pressure, the bcc solid is the most stable solid. This represents a difference with the KF (Ref. 4) model studied by Romano et al. ${ }^{36}$ for which the bcc and diamond solids exhibit the same energy (both maximize the number of bonds per particle) and so they are degenerate at zero temperature and at zero pressure. At zero temperature and pressures above zero, the more stable phase will be that with lower enthalpy. As the molar volume of the bec solid is lower than that for diamond, the $p V$ term is lower for the bec solid, and therefore the bcc solid is again more stable than the diamond structure. In summary, for our model, at zero temperature the diamond structure becomes more stable than the bcc solid only at negative pressures. At zero pressure and finite temperatures, however, it is probable that the diamond structure has a higher vibrational entropy because interactions between the two sublattices are likely to reduce the vibrational entropy of the bcc solid, i.e., the atoms in the bcc solid have less "room" to vibrate because of the presence of the other sublattice. Therefore, it is possible that the diamond structure could be stabilized if the entropy term, which is expected to be somewhat higher in the diamond structure, overcomes the advantage in the potential energy of the bcc solid.

For completeness, the high pressure region of the phase diagram will also be studied. Similar to what has been found for octahedral particles, ${ }^{35}$ it is expected that at high pressures a face-centered-cubic (fcc) solid is the most stable phase. For tetrahedral particles, it is not possible to align each of the four patches with a nearest neighbor. However, an ordered fcc structure (fcc-o) can be obtained by starting from the bcc lattice described above and then stretching one of the edges of the unit cell from $a$ to $a \times \sqrt{2}$ so that each one of the four patches will be pointing to four of the 12 nearest neighbors, but the alignment will not be perfect [Fig. 1(c)]. This structure has a somewhat higher energy than the diamond or the bcc lattices. It is expected to become the stable phase above some given pressure, where this high density structure will be favored by its lower enthalpy (the $p V$ term will compensate for the disadvantage in the potential energy).

At high temperatures, where the kinetic energy is high enough to overcome the attractive interactions, it is likely that the ordered fcc will transform into a plastic crystal in which the centers of mass of the particles are arranged in a fcc lattice but where the particles are free to rotate. The plastic crystal will be denoted fcc-d.

\section{Details of the simulations}

NpT Monte Carlo (MC) simulations were used for both the fluid and the solid phases. Typically, about $200000 \mathrm{MC}$ cycles (plus another $100000 \mathrm{MC}$ cycles for equilibration) were used for the fluid phase, whereas $50000 \mathrm{MC}$ cycles (plus $50000 \mathrm{MC}$ cycles for equilibration) were enough for the solid phases. Each MC cycle consisted of $N$ attempts to translate or rotate a particle (with $N$ being the number of particles in the system) plus one attempt to change the volume. The maximum translational and rotational displacements were adjusted to obtain a $40 \%$ acceptance probability and the maximum volume displacement was adjusted to obtain a $30 \%$ acceptance probability. The number of particles used in the simulations was 512 for the diamond crystal, 432 for the bcc lattice, 500 for the fcc solid, and 432 for the fluid phase. These numbers are chosen so that the crystal structures are commensurate with the simulation boxes.

The computation of the phase diagram requires the calculation of free energies. As the methods used to compute free energies in this work have been previously described in detail, ${ }^{48-50}$ only a brief summary will be given here. For the fluid, the free energy was calculated by thermodynamic integration with the ideal gas as a reference state. ${ }^{51}$ Between 10 and 20 states were used in the integration. For the solid phases, we used the recently proposed Einstein molecule approach, ${ }^{48-50}$ which is a variant of the Einstein crystal method of Frenkel and Ladd. ${ }^{52,53}$ In this method, the free 
TABLE I. Coexistence points for the 12-6 model obtained using thermodynamic integration together with the Helmholtz free energies given as supplementary material (Ref. 60). Uncertainties in the potential energy per particle $u^{*}$ are smaller than 0.01 .

\begin{tabular}{lccccccr}
\hline \hline Phase 1 & Phase 2 & $T^{*}$ & $p^{*}$ & $\rho_{1}^{*}$ & $u_{1}^{*}$ & $\rho_{2}^{*}$ & \multicolumn{1}{c}{$u_{2}^{*}$} \\
\hline Fluid & bcc & 0.100 & $0.0005(2)$ & $0.0005(2)$ & -0.07 & $0.882(1)$ & -1.64 \\
Diamond & bcc & 0.100 & $-0.003(2)$ & $0.441(1)$ & -1.62 & $0.882(1)$ & -1.64 \\
bcc & fcc-o & 0.100 & $4.06(5)$ & $1.068(1)$ & -1.44 & $1.179(1)$ & -1.00 \\
Fluid & bcc & 0.150 & $0.21(5)$ & $0.554(1)$ & -0.35 & $0.873(1)$ & -1.43 \\
Fluid & fcc-d & 0.500 & $5.91(5)$ & $0.985(1)$ & 0.02 & $1.082(1)$ & -0.04 \\
Fluid & fcc-d & 1.000 & $13.1(3)$ & $1.036(1)$ & 0.58 & $1.136(1)$ & 0.42 \\
\hline \hline
\end{tabular}

energy of the solid is calculated by Hamiltonian integration with the reference state being an Einstein molecule (i.e., an Einstein crystal in which one of the molecules, e.g., molecule 1 , does not vibrate) with the same lattice as the real solid. As we are considering anisotropic particles, besides the harmonic springs that bind the center of mass of each particle to a lattice position, an orientational field that keeps the particles with the right orientation is also needed. It is convenient to choose an orientational field with the same symmetry as the model under study. ${ }^{48,54}$ The orientation of each particle in the reference structure is defined by two unitary vectors $\mathbf{a}_{\mathbf{0}}$ and $\mathbf{b}_{\mathbf{0}}$ (nonorthogonal) parallel to two specified patches. For the tetrahedral particles (that exhibit $\mathrm{T}_{d}$ symmetry), the reference system will be

$$
\begin{aligned}
U_{\text {Ein-mol }} & =U_{\text {trans }}+U_{\text {orient }} \\
& =\sum_{i=2}^{N} \lambda_{t}\left(\mathbf{r}_{i}-\mathbf{r}_{i, 0}\right)^{2}+\sum_{i=1}^{N} \lambda_{o}\left[\sin ^{2}\left(\Psi_{a, i}\right)+\sin ^{2}\left(\Psi_{b, i}\right)\right],
\end{aligned}
$$

where $\lambda_{t}$ and $\lambda_{o}$ are the coupling parameters, $\mathbf{r}_{i}$ is the instantaneous position of the center of mass of molecule $i$, and $\mathbf{r}_{i, 0}$ is its equilibrium position. $\Psi_{a, i}$ is the angle formed by the closest patch in the instantaneous orientation of molecule $i$ and the vector $\mathbf{a}_{\mathbf{0}}$ in the reference structure, and $\Psi_{b, i}$ is defined analogously. Note that the second sum in Eq. (4) runs over all the particles, i.e., all molecules are allowed to rotate. ${ }^{48,49}$ The free energy of the reference system and the free energy difference between the reference system and the solid was evaluated by using the procedure described in Refs. 48 and 50.

Once the free energy is known at a given thermodynamic state, the free energy can be computed at other states by thermodynamic integration. ${ }^{48,51}$ Coexistence points were calculated by imposing the conditions of chemical equilibrium, i.e., equal temperature, pressure, and chemical potential. Starting from the coexistence points calculated by free energy calculations, Gibbs-Duhem integration ${ }^{55,56}$ with a fourth order Runge-Kutta algorithm ${ }^{57}$ was used to trace the coexistence lines.

The melting point of the diamond and bcc solids was also calculated by using the direct coexistence method. ${ }^{35,58,59}$ We follow the same procedure as that described in Ref. 35 . In this method, simulations of a fluid-solid interface are performed. For the fluid-diamond interface, the initial configuration contained 512 solid particles (i.e., $4 \times 4 \times 4$ unit cells) and 512 fluid particles. The fluid-bcc interface contained 432 solid particles (i.e., $6 \times 6 \times 6$ unit cells) and another 432 fluid particles. Finally, in the fluid-fcc-d interface there was a crystalline block of 500 particles (i.e., $5 \times 5 \times 5$ unit cells) plus another 500 fluid particles. The interfaces were generated as in Ref. 35. The coexistence point was then calculated by performing $N p T$ MC simulations at a given temperature and at different pressures. Monitoring the evolution of the internal energy or the density, it is possible to bracket the coexistence pressure at the simulated temperature. Alternatively, at a given pressure, simulations can be performed at various temperatures to bracket the coexistence temperature at that pressure.

\section{RESULTS}

Let us start with the results for the long-ranged 12-6 LJ tetrahedral model. Before presenting the computed phase diagram, we first consider the fluid-fluid phase equilibrium. In a previous study of octahedral particles using the same model potential as the one used here, it was found that for a patch width of $\sigma_{\mathrm{pw}}=0.3 \mathrm{rad}$, fluid-fluid phase separation was metastable with respect to solidification. ${ }^{35}$ As shown in previous work, ${ }^{4}$ the fluid-fluid phase separation moves to lower temperatures as the surface coverage of the patches diminishes. From this result, it follows that for the same patch width, the fluid-fluid phase separation for the tetrahedral model (four patches) occurs at a lower temperature than that of the octahedral model (six patches). As fluid-fluid phase separation was already metastable for the octahedral model at a patch width of $\sigma_{\mathrm{pw}}=0.3 \mathrm{rad},{ }^{35}$ it is likely that it is also metastable for the tetrahedral model. For this reason, studies of fluid-fluid phase separation were not attempted in this work, although it would be interesting to study the emergence of an equilibrium liquid phase at larger $\sigma_{\mathrm{pw}}$ in future work.

We focus now on the fluid-solid and solid-solid phase separation. Helmholtz free energies were calculated for all the considered solid phases at some specified thermodynamic states (this data is provided as supplementary material ${ }^{60}$ ). These free energy calculations were subject to thermodynamic consistency checks. Once the free energy is known at a particular thermodynamic state, coexistence points can be obtained by thermodynamic integration (see Table I). Let us consider the results for $T^{*}=0.1$. At this temperature, there is a phase transition at almost zero pressure from a very low density fluid to the bcc solid, which upon increasing the pres- 


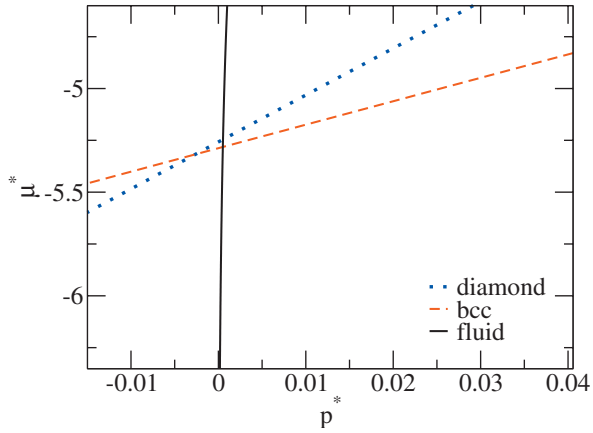

FIG. 2. Chemical potential $\left(\mu^{*}=\mu / k T\right)$ as a function of pressure for the fluid phase and the diamond and bcc solids for the 12-6 LJ model along the $T^{*}=0.1$ isotherm

sure transforms into the orientationally ordered fcc-o phase. The bcc phase is stabilized with respect to the fluid and the fcc-o solid by a low average potential energy. The fcc-o solid has an appreciably higher energy than the bcc (see Table I), but for sufficiently high pressures, the fcc-o becomes more favorable due to its higher density and entropy (which is probably due to the greater orientational freedom that arises because the patches cannot perfectly align with all the nearest neighbors).

We now consider the stability of the diamond structure at this temperature. The chemical potentials for the fluid phase, and the diamond and bec solids along the $T^{*}=0.1$ isotherm are shown in Fig. 2. Our results predict that the diamond structure only becomes more stable than the bcc solid at slightly negative pressures (see Table I) in a region of the phase diagram where the fluid phase is more stable than both the diamond and the bcc solids. We should note that as the difference in pressure between the fluid-bcc and the diamond-bcc coexistence points is so small at this temperature, the accuracy of the present calculations does not allow us to totally rule out the possibility that these transitions occur in a different order. However, even if this was the case, the diamond crystal would at most only be marginally stable at very low pressures at this temperature. Moreover, as mentioned in Sec. II B, the metastability of the diamond structure is not unexpected because the bcc solid shows a slightly lower energy and also a lower $p V$ term due to its higher density. Therefore, the diamond structure could only be stabilized if it would exhibit a higher vibrational entropy than the bcc solid to overcome the lower enthalpy of the latter. It is reasonable to think that diamond might have a somewhat higher entropy because it is likely that in the bcc solid the movement of the particles is somewhat impeded by interactions between the two diamond sublattices. If correct, the diamond structure should then gain stability with respect to the bcc solid as the temperature is increased and so might even become the thermodynamically stable phase at higher temperature. To check this possibility, the fluid-bcc and the diamond-bcc coexistence lines were calculated using GibbsDuhem integration. As shown in Fig. 3, the diamond crystal indeed gains some stability with respect to the bcc solid as the temperature increases, although the effect is relatively small. At higher temperatures, the diamond/bcc coexistence

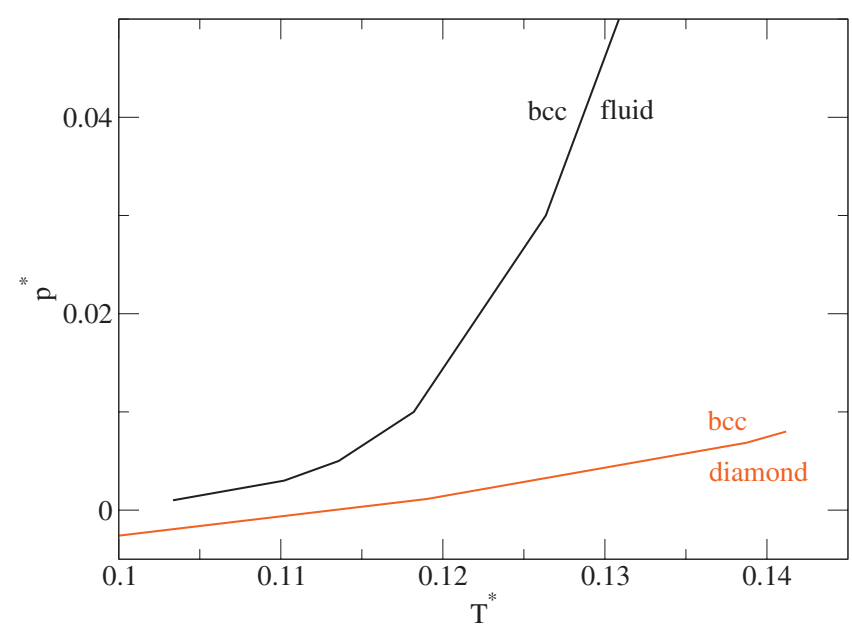

FIG. 3. Fluid-bcc and diamond-bcc coexistence lines for the 12-6 LJ model obtained from Gibbs-Duhem simulations. Above $T^{*}=0.14$, the diamond solid is not mechanically stable.

occurs at slightly positive pressures. However, this happens in a region where the most stable phase is the fluid, i.e., after the sublimation of the bec solid.

The absence of a region in the phase diagram in which the diamond solid is the most stable phase differs from recent calculations of the phase diagram of tetrahedral particles performed by Romano et al. ${ }^{36}$ However, these authors used a different model potential to describe the interactions between the tetrahedral particles and most likely the differences between the present work and that of Romano et al. are due to the use of a different model potential. Indeed, Vega and Monson using the primitive model of water (PMW) (Ref. 61) that bears some resemblance to the KF model also found that the diamond lattice was thermodynamically stable. ${ }^{62}$ The PMW particles are also hard spheres with four patches in a tetrahedral arrangement, whose interactions are modeled using square-well potentials. However, in the PMW there are two inequivalent types of patches, and only patches of different type interact. The possible origin of the differences between our work and that of Romano et al. will be discussed in more detail later.

Starting from the coexistence points given in Table I the whole coexistence lines were obtained using the GibbsDuhem integration method. Some coexistence points calculated by this method are given as supplementary material. ${ }^{60}$ As a test, the melting points of the solid phases that are in coexistence with the fluid (i.e., the bcc solid and the fcc plastic crystal) were also computed using the direct coexistence method. The melting points obtained by this route are shown in Table II. As can be seen, free energy calculations and the direct coexistence method give results that are consistent within statistical uncertainty.

The complete phase diagram for the tetrahedral model with a patch width $\sigma_{\mathrm{pw}}=0.3 \mathrm{rad}$ is shown in Fig. 4. All the solids considered, except diamond, are stable over a region of the phase diagram. At low temperatures, the fluid freezes into the bec crystal, which upon compression is destabilized with respect to the fcc-o solid. The fcc-o structure transforms into a plastic crystal (fcc-d) at approximately $T^{*}=0.16$. This order-disorder transition is a first order transition; it exhibits 
TABLE II. Coexistence points for the 12-6 model obtained using the direct coexistence method. For comparison, the results from free energy calculations are also given.

\begin{tabular}{|c|c|c|c|c|c|}
\hline \multirow[b]{2}{*}{ Phase 1} & \multirow[b]{2}{*}{ Phase 2} & \multicolumn{2}{|c|}{ Direct coexistence } & \multicolumn{2}{|c|}{ Free energy calculations } \\
\hline & & $T^{*}$ & $p^{*}$ & $T^{*}$ & $p^{*}$ \\
\hline Fluid & bcc & $0.14(1)$ & 0.21 & 0.15 & $0.21(2)$ \\
\hline Fluid & fcc-d & 0.50 & $6.0(1)$ & 0.50 & $5.91(2)$ \\
\hline
\end{tabular}

a discontinuity both in the energy and in the density. As this region of the phase diagram is not the focus of this work, the temperature at which the order-disorder transition occurred at a given pressure was estimated simply as the midpoint of the hysteresis loops in the variation of the energy and density with temperature upon heating and quenching. The phase diagram exhibits a triple point at $T^{*}=0.201$ and $p^{*}=2.03$, at which the fluid, the bcc and the fcc-d solids coexist. Above this temperature, the fluid freezes into the fcc-d plastic crystal.

The phase diagram of the tetrahedral particles is simplified with respect to that of the six-patch octahedral particles that we computed previously. ${ }^{35}$ Additional features for the octahedral system include the stabilization of a low density
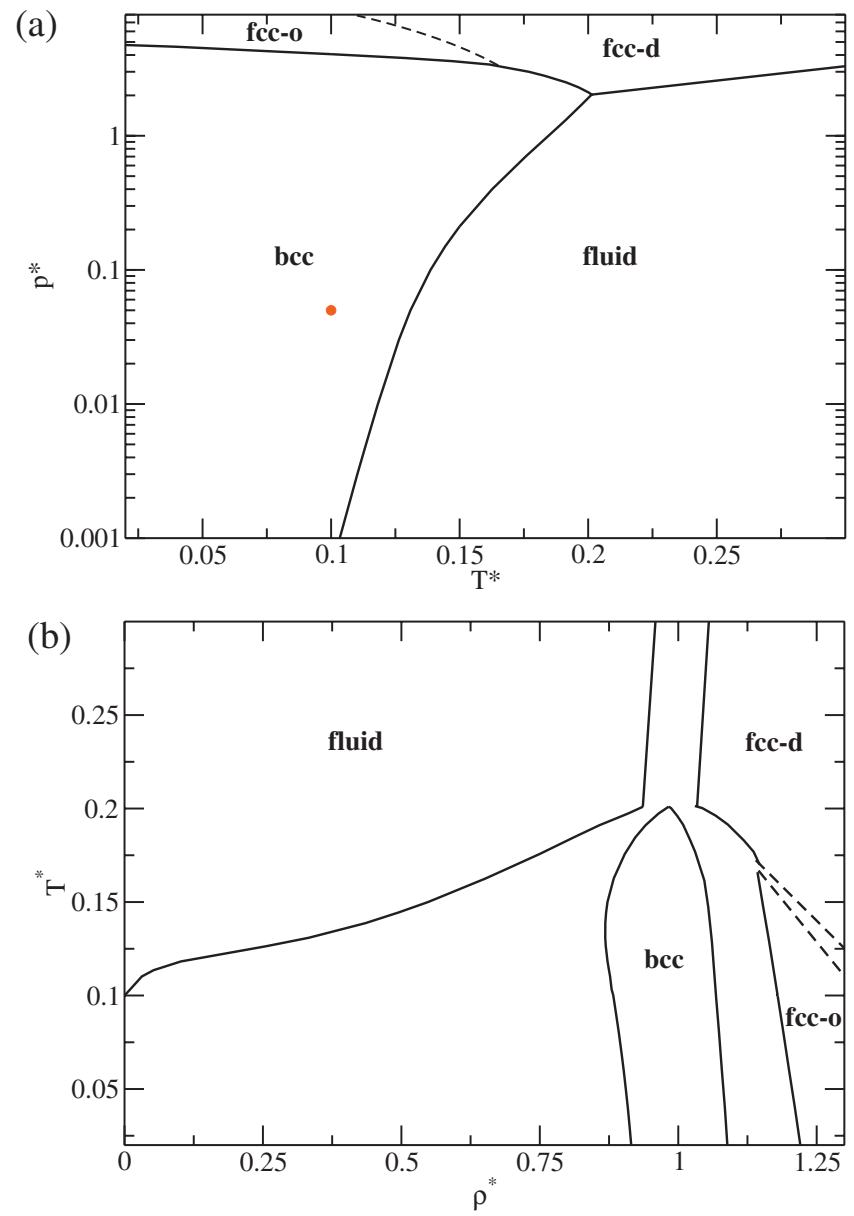

FIG. 4. Phase diagram of model patchy particles with tetrahedral symmetry for the 12-6 model and a patch width of $\sigma_{\mathrm{pw}}=0.3 \mathrm{rad}$ as a function of (a) pressure and temperature, and (b) temperature and density. The dot in (a) indicates the thermodynamic state point at which direct coexistence simulations of the fluid-diamond and fluid-bcc interface were performed to study the growth behavior of these solids. crystal (a simple-cubic solid) and reentrant behavior for the coexistence lines between the fluid and simple-cubic solid and between the bcc and fcc crystals. Another difference between the two models is that the bcc solid is almost incompressible at zero temperature for the octahedral model, whereas for the tetrahedral model, the bcc can be compressed from $\rho^{*}=0.915$ up to $\rho^{*}=1.225$, which correspond to nearestneighbor distances of $1.124 \sigma_{\mathrm{LJ}}$ and $1.061 \sigma_{\mathrm{LJ}}$, respectively. This means that the bcc solid can be compressed considerably from the minimum energy structure (i.e., that for which nearest neighbors are located at the distance of the minimum of the potential $1.123 \sigma_{\mathrm{LJ}}$ ). The different behavior is because the interpenetration of the two diamond sublattices does not have an energy penalty but the interpenetration of two simple-cubic sublattices does.

However, there are also strong similarities between the two phase diagrams and quantitative comparisons can be made. For example, the bcc phase is stable up to $T^{*}=0.336$ for the octahedral particles, whereas for the tetrahedral particles, it is stable up to $T^{*}=0.201$. As tetrahedral particles have only four patches whereas octahedral have six patches, one would expect that the maximum temperature for which the bcc solid is stable for the tetrahedral particles should be roughly two thirds of that for octahedral particles, which is in agreement with our results. Note that in both cases the bcc solid is stabilized by its low internal energy achieved because the patches can directly point at the neighboring particles.

As already noted, the phase diagram obtained here is somewhat different from the phase diagram recently reported by Romano et al. for similar patchy tetrahedral particles. ${ }^{36} \mathrm{In}$ contrast to our results, these authors found that the diamond crystal is stable over a region of the phase diagram. What is the origin of these differences? Romano et al. used a different model potential from the one studied here. In particular, they considered the KF model in which particles are described as hard spheres with some attractive patches at the surface modeled by a square-well potential in both relative orientation and interparticle distance. ${ }^{4}$ Taking a fixed value for the patch width of $0.4 \mathrm{rad}$, these authors calculated the phase diagram for different ranges of the potential (from $0.03 \sigma_{\mathrm{HS}}$ to $0.24 \sigma_{\mathrm{HS}}$, where $\sigma_{\mathrm{HS}}$ is the diameter of the hard spheres) and found that the bcc solid phase is destabilized with respect to the diamond structure and the fluid phase as the range of the potential decreases. ${ }^{63}$ Therefore, this suggests that the diamond structure might become stabilized in our model if the range of the potential was decreased.

We checked this hypothesis by also calculating the phase diagram for a shorter-ranged model, where the LJ model was 
TABLE III. Coexistence points for the 20-10 model obtained using thermodynamic integration together with the Helmholtz free energies given in the supplementary material (Ref. 60). Uncertainties in the densities $\rho^{*}$ are of the order of 0.001 and in the potential energy per particle $u^{*}$ are smaller than 0.01 .

\begin{tabular}{lccccccc}
\hline \hline Phase 1 & Phase 2 & $T^{*}$ & $p^{*}$ & $\rho_{1}^{*}$ & $u_{1}^{*}$ & $\rho_{2}^{*}$ & $u_{2}^{*}$ \\
\hline Fluid & Diamond & 0.10 & $0.001(1)$ & 0.010 & -0.04 & 0.515 & -1.65 \\
Diamond & bcc & 0.10 & $0.02(2)$ & 0.516 & -1.65 & 1.025 & -1.67 \\
bcc & fcc-o & 0.10 & $5.49(6)$ & 1.131 & -1.57 & 1.238 & -1.07 \\
Fluid & bcc & 0.13 & $0.09(1)$ & 0.404 & -0.31 & 1.013 & -1.55 \\
bcc & fcc-o & 0.13 & $5.22(6)$ & 1.126 & -1.48 & 1.227 & -1.00 \\
Fluid & fcc-d & 0.50 & $5.70(6)$ & 0.962 & -0.02 & 1.065 & -0.07 \\
\hline \hline
\end{tabular}

replaced by a generalized LJ potential with exponents of 20 and 10 rather than 12 and 6 . The free energies at some selected thermodynamic states are given as supplementary material. ${ }^{60}$ Coexistence points calculated from these data are shown in Table III, and coexistence lines obtained from Gibbs-Duhem simulations are also given as supplementary material. ${ }^{60}$ The melting points of the solid phases in coexistence with the fluid were checked by also performing direct coexistence simulations. The agreement between the two routes was satisfactory (see Table IV). The complete phase diagram is shown in Fig. 5. Besides the bcc and fcc solids found for the 12-6 model, the diamond crystal is now stable over a region of the phase diagram. For sufficiently low pressures, the diamond structure is stabilized over the bcc at finite temperatures. As can be seen in the temperature and density phase diagram, the diamond structure is only stable for a very narrow range of densities, which is a consequence of its low compressibility. An enlarged view of the pressure and temperature phase diagram (see Fig. 6) shows that the coexistence between the diamond and bcc solid phases occurs at negative pressures for temperatures below about $T^{*}$ $=0.03$, which means that the bcc is the solid stable phase at very low temperatures. As mentioned in Sec. II B, at zero temperature and pressure the bcc phase is more stable than diamond and the same occurs at positive pressures because the former has a lower energy.

As for the bcc solid, its region of coexistence moves to higher densities because the minimum of the potential moves to shorter distances. At temperatures close to zero, the bcc solid is stable for densities between $\rho^{*}=1.051$ and $\rho^{*}$ $=1.146$, which correspond to nearest-neighbor distances of $1.072 \sigma_{\mathrm{LJ}}$ (the distance of the minimum of the $20-10 \mathrm{LJ}$ model) and $1.043 \sigma_{\mathrm{LJ}}$, respectively. As before, the bcc solid can compress considerably before losing its stability with respect to the fcc solid. It can also be seen that the bcc solid gains some stability with respect to the fcc solid as the range of the potential shortens (i.e., the bcc-fcc phase transition moves to higher pressures as the range decreases). Again the fcc solid exhibits an order-disorder transition at $T^{*} \approx 0.17$. The thermodynamic states of the two triple points calculated for the 20-10 model are given in Table V.

We have seen that, as for the KF model, the diamond structure is stabilized with respect to the bcc solid when the range of the interactions decreases. But why is this so? A quick route to obtain information about the phase diagram of a given model is by calculating the properties of the competing solid phases at zero temperature. ${ }^{64}$ At zero temperature, the condition of chemical equilibrium is given by the equality of enthalpy of the phases in coexistence,

$$
\begin{aligned}
& U_{I}\left(p_{\text {eq }}, T=0\right)+p_{\text {eq }} V_{\mathrm{I}}\left(p_{\text {eq }}, T=0\right) \\
& \quad=U_{\text {II }}\left(p_{\text {eq }}, T=0\right)+p_{\text {eq }} V_{\text {II }}\left(p_{\text {eq }}, T=0\right) .
\end{aligned}
$$

Therefore, phase transitions can be located at zero temperature without computing free energies, just by calculating the density and potential energy of both phases in coexistence. Assuming that the change of internal energy and the change of volume between the two phases are independent of pressure, the coexistence pressure at zero temperature can be approximately calculated using ${ }^{65}$

$$
p_{\text {eq }}=-\frac{\Delta U(p=0, T=0)}{\Delta V(p=0, T=0)} .
$$

Using this expression, we have calculated the coexistence pressure at zero temperature between the diamond and bcc solids for the models studied in this work. The densities and energies at zero temperature and pressure were estimated by performing simulations at temperatures between $T^{*}=0.04$ and $T^{*}=0.005$ and linearly extrapolating these data to $T^{*}=0$ (see Fig. 7). The densities and internal energies obtained using this procedure are given in Table VI. It is found that, as expected (see Sec. II B), for both models the diamond-bcc transition occurs at negative pressures. The coexistence pressure is about $p^{*}=-0.009$ for the $12-6$ model and about

TABLE IV. Coexistence points for the 20-10 model obtained using the direct coexistence method. For comparison, the results from free energy calculations are also given.

\begin{tabular}{lcccccc}
\hline \hline & & \multicolumn{2}{c}{ Direct coexistence } & & \multicolumn{2}{c}{ Free energy calculations } \\
\cline { 3 - 4 } \cline { 6 - 7 } Phase 1 & Phase 2 & $T^{*}$ & $p^{*}$ & & $T^{*}$ & $p^{*}$ \\
\hline Fluid & bcc & 0.15 & $0.30(1)$ & & 0.15 & 0.30 \\
Fluid & fcc-d & 0.30 & $3.2(1)$ & & 0.30 & 3.22 \\
\hline \hline
\end{tabular}


(a)

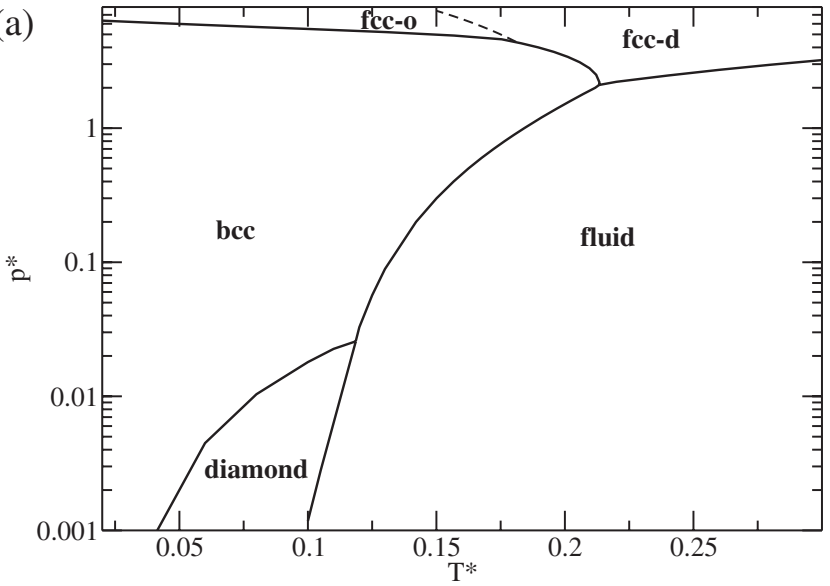

(b)

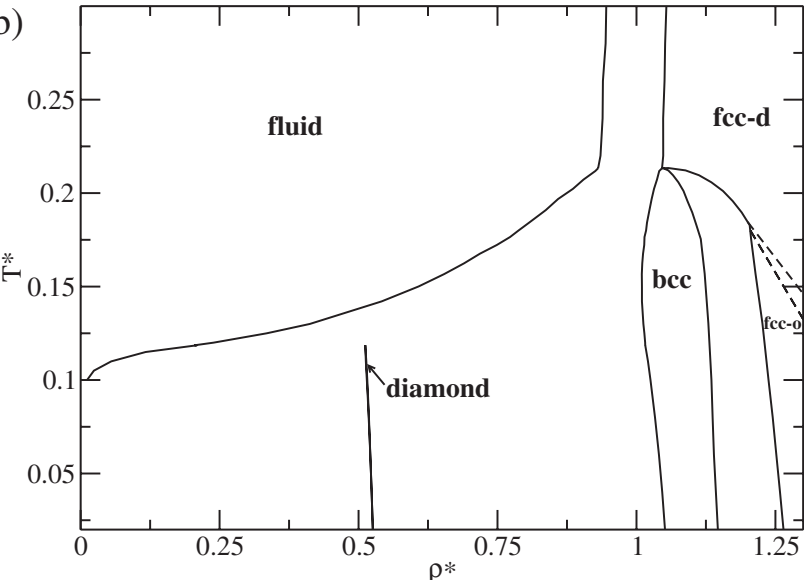

FIG. 5. Phase diagram of model patchy particles with tetrahedral symmetry for the 20-10 model and a patch width of $\sigma_{\mathrm{pw}}=0.3 \mathrm{rad}$ as a function of (a) pressure and temperature, and (b) temperature and density.

$p^{*}=-0.0003$ for the $20-10$ model. The less negative coexistence pressure for the shorter-ranged potential is a consequence of the significantly smaller difference in energy between the two crystals for the 20-10 model. This indicates that, even at zero temperature, the diamond solid is stabilized by decreasing the range of the interactions. It also provides a possible recipe to predict the stability of the diamond solid:

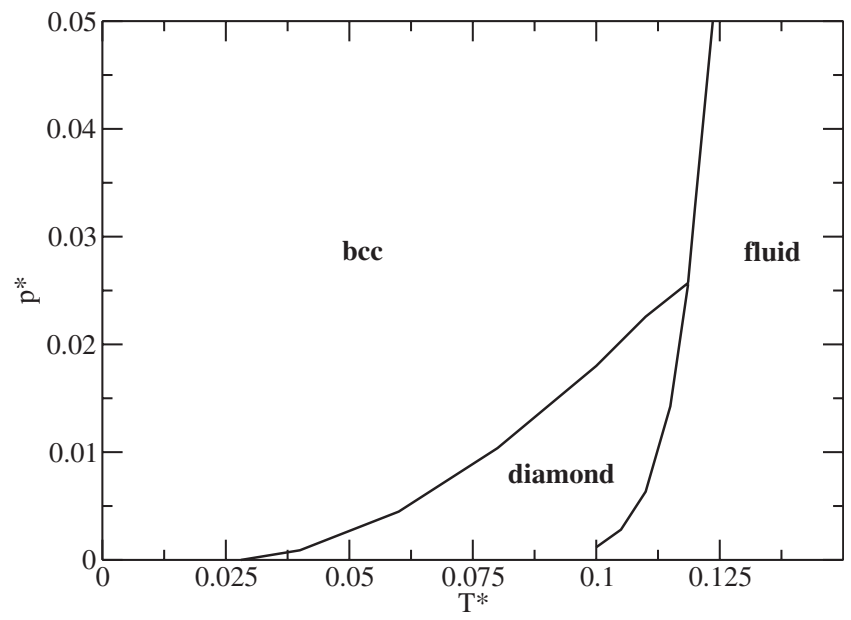

FIG. 6. Enlarged view of the low temperature and low pressure region of the phase diagram for the 20-10 model.
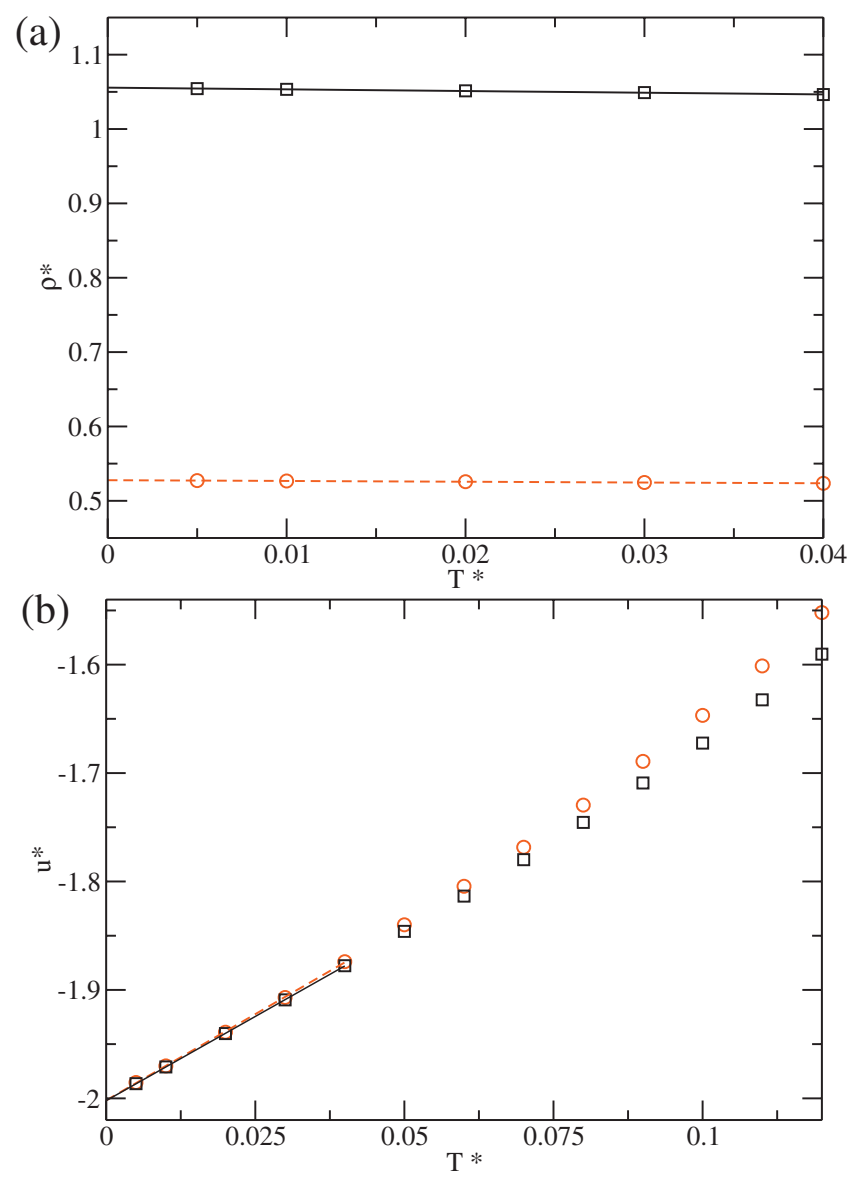

FIG. 7. (a) Densities and (b) energies of the bcc solid (squares and solid line) and of the diamond solid (circles and dashed line) along the isobar $p^{*}=0$ for the $20-10$ model.

the less negative the coexistence pressure, the higher the probability of stabilizing the diamond solid. It is interesting to note that the behavior of our model and the KF model at zero temperature will be different. In the KF model, the diamond and the bcc crystals exhibit the same energy at zero temperature, and so the phase transition occurs exactly at zero pressure, i.e., for any positive pressure, the bcc solid will be more stable than the diamond, which will become more stable than the bcc only at negative pressures.

To further understand the origin of the stabilization of the diamond structure at finite temperature as the range of the interactions decreases, we have calculated the different contributions to the chemical potential for both the bcc and diamond solids along the $p^{*}=0.01$ isobar, a pressure at which diamond is stable over some temperature range for the 20-10 model. The difference between the chemical potential of the bcc and the diamond solids $\left(\Delta \mu=\mu^{\mathrm{bcc}}-\mu^{\text {diamond }}\right)$ and its three contributions $(\Delta \mu / k T=\Delta U / N k T+\Delta(p V) / N k T$ $-\Delta S / N k)$ are shown in Fig. 8. The chemical potential is computed by thermodynamic integration along the isotherm $p^{*}=0.01$ starting from the free energy at $T^{*}=0.10$.

Results for both the long-ranged 12-6 LJ model and the shorter-ranged 20-10 model are shown. At this pressure, $p^{*}$ $=0.01, \Delta \mu / k T$ is negative over all the temperature range for the 12-6 model (i.e., the bcc solid is more stable than diamond), and passes from negative to positive at $T^{*} \approx 0.08$ for 
TABLE V. Thermodynamic properties of the triple points found for the 20-10 model.

\begin{tabular}{lccccccc}
\hline \hline Phase 1 & Phase 2 & Phase 3 & $T^{*}$ & $p^{*}$ & $\rho_{1}^{*}$ & $\rho_{2}^{*}$ & $\rho_{3}^{*}$ \\
\hline Fluid & Diamond & bcc & 0.119 & 0.026 & 0.205 & 0.512 & 1.016 \\
Fluid & bcc & fcc & 0.213 & 2.10 & 0.931 & 1.046 & 1.046 \\
\hline \hline
\end{tabular}

the 20 -10 model (i.e., for temperatures below $T^{*} \approx 0.08$, the bcc solid is most stable but for temperatures above this the diamond structure is most stable). Analyzing the contributions to the chemical potential, it can be seen that the $\Delta(p V) / N k T$ term is practically independent of the temperature (for the range of temperatures considered) and, at this pressure, its value is very similar for the two models studied. The important term for understanding the thermal stabilization of the diamond structure is $\Delta S$. In the harmonic approximation, the vibrational entropy is given by

$$
S_{\mathrm{vib}}=(6 N-3) k\left(\ln \left(\frac{k T}{h \bar{\nu}}\right)+1\right),
$$

where $\bar{\nu}$ is the geometric mean vibrational frequency. Note that in a classical statistical mechanics formalism (as is appropriate for the simulations considered in this work), the entropy tends to minus infinity at zero temperature. However, the difference in entropy between two phases can remain finite. In particular, it follows that, again in the harmonic approximation,

$$
\Delta S_{\mathrm{vib}}=(6 N-3) k \ln \left(\frac{\bar{\nu}_{\text {diamond }}}{\bar{\nu}_{\text {bcc }}}\right) .
$$

As Fig. 8 shows, $\Delta S$ approaches zero as the temperature is decreased. The vibrational frequencies are related to the curvature of the potential energy surface at the minimum corresponding to the crystal. At this minimum, the two sublattices of the bcc crystal interact only very weakly, and so the vibrational frequencies are essentially the same for the two crystals, except for the three modes corresponding to the displacement of the two sublattices with respect to each other in the bcc crystal. Hence, $\Delta S(T=0) \approx 0$.

Equation (8) also implies that $\Delta S_{\text {vib }}$ will not vary with temperature if the vibrations are harmonic. However, as is clear from Fig. 7, the variation of the potential energy with temperature deviates from the linearity expected for harmonic vibrations, and it is noticeable that this deviation is more pronounced for the diamond lattice, i.e., the vibrations in the diamond lattice are more anharmonic. As the magnitude of the vibrations increases with temperature, the presence of another sublattice becomes an increasing constraint on the thermal motion in the bcc crystal. By contrast, the empty space in the diamond lattice allows the magnitude of the vibrations to increase more rapidly than that for a harmonic system. It is this greater vibrational entropy that drives the thermal stabilization of the diamond phase. There is one more subtlety: the increase of $-\Delta S$ with temperature is partially offset by a corresponding decrease in $\Delta U$ because the greater vibrational entropy available as the energy increases causes the energy to increase more rapidly than for harmonic vibrations. This relationship between $\Delta S$ and $\Delta U$ is, of course, inherent in the formulas: $(\partial S / \partial T)_{P}=C_{p} / T$ and $(\partial U / \partial T)_{P}=C_{p}$.

This analysis leaves one question: why is this thermal stabilization of the diamond crystal more pronounced for the shorter-range potential. First, the zero-temperature difference in potential energy between the two crystals is reduced. Second, as we noted earlier the density difference between the bcc crystals when at its potential energy minimum and when the repulsive cores start to overlap is smaller for the 20-10 model. Hence, the magnitude of the vibrations required for the two sublattices to begin to interact significantly is smaller. The potential effect of this difference is reduced because the magnitude of the bond-stretching vibrations is also reduced for the 20-10 model because the potential is stiffer as a function of distance. However, the patch width is the same for the two models, and hence the magnitude of the angular vibrations will be similar for the two models, and it is the effect of the reduced "room" on the angular vibrations in the bcc crystal that leads to the enhanced entropic stabilization of the diamond structure for the shorter-ranged model.

In summary, our results show significant similarities with those of Romano et al. ${ }^{36}$ First, we find that at low pressure, the bcc and diamond crystals have very similar free energies. Second, we found that the diamond solid is stabilized as the range of the potential decreases. However, the main difference with their results is that the region of diamond stability is significantly reduced, and that for a sufficiently long-ranged potential, the diamond crystal is never thermodynamically the most stable phase. This difference is most likely due to the different shapes of the potential wells for the two models.

In the KF model, the patches are modeled as square wells, and the flat-bottomed nature of these wells means that if no bonds are broken (in the work of Vega and Monson using the PMW model they indeed found that in both the diamond and bec solids breaking of bonds was a rare event $^{62}$ ), the configuration space available to the solid at a given density is independent of temperature. Thus, there is an entropy term associated with the rattling of the molecules in these square wells even at zero temperature. Furthermore, this entropy term will favor the diamond crystal because of

TABLE VI. Density and potential energy at zero temperature and pressure for the diamond and bcc solids for the two studied model potentials.

\begin{tabular}{cccc}
\hline \hline Model & Solid & $\rho^{*}$ & $u^{*}$ \\
\hline \multirow{2}{*}{$12-6$} & Diamond & 0.4599 & -1.9765 \\
& bcc & 0.9206 & -1.9867 \\
$20-10$ & Diamond & 0.5277 & -2.0019 \\
& bcc & 1.0557 & -2.0021 \\
\hline \hline
\end{tabular}




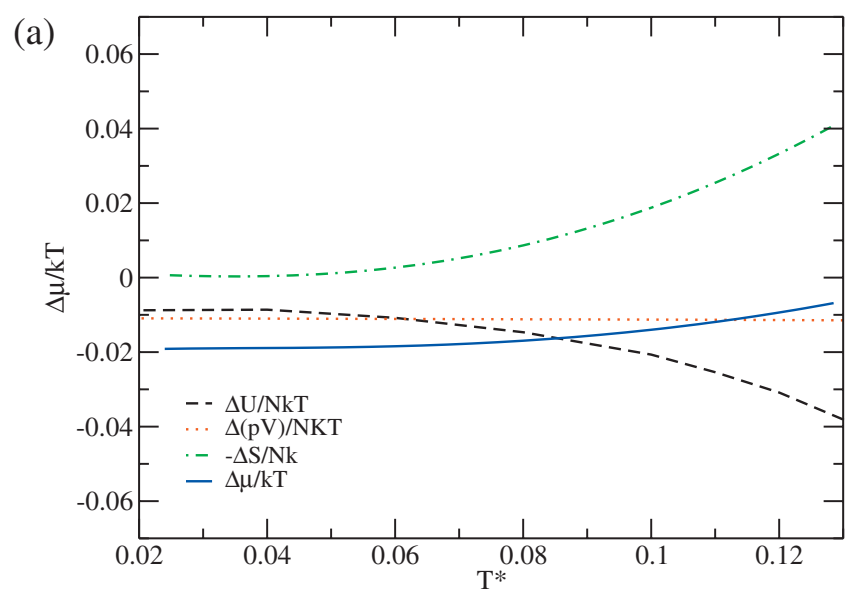

(b)

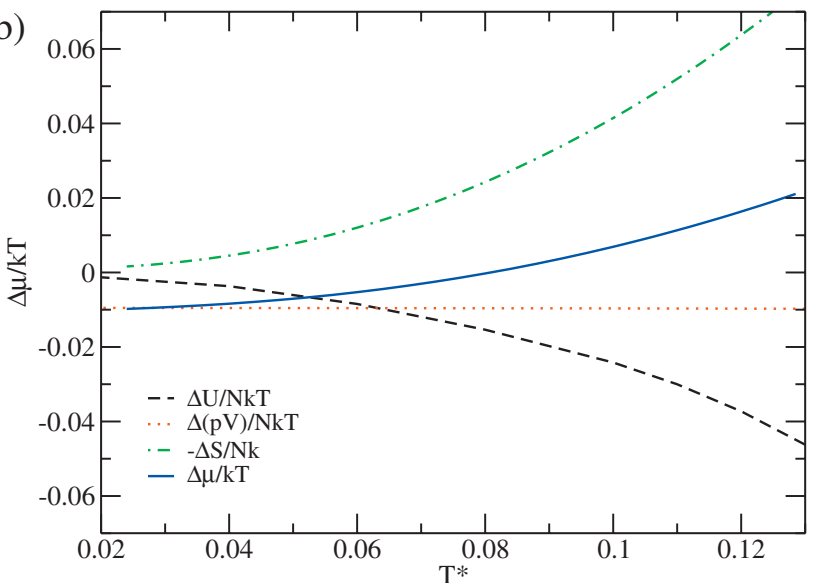

FIG. 8. Chemical potential difference between the bcc and diamond solids along the $p^{*}=0.01$ isobar for (a) the 12-6 model and (b) the 20-10 model. The contributions of potential energy, $p V$, and entropy to the chemical potential are also given.

the reduction in the configuration space available to the bcc solid due to interactions between the two sublattices. By contrast, for our patchy LJ model, the system becomes localized in the potential energy minimum corresponding to the respective solid at zero temperature because all vibrations come with a potential energy cost. Only as the temperature increases, and hence the amplitude of the vibrational motion increases, does a difference in vibrational entropy favoring diamond become apparent.

Now that the phase diagram for our patchy particles is known, it would also be interesting to perform nucleation studies to investigate which solid structure nucleates from the fluid at different thermodynamic states. This is by no means a trivial question. For example, a significant number of systems have been shown to follow Ostwald's step rule $;{ }^{66-68}$ namely, that a fluid does not crystallize in the most thermodynamically stable phase if there is an alternative that is separated from the fluid by a lower free energy barrier. Given the small free energy differences between the bcc and diamond crystals at low pressure, it would not be so surprising if the selection of crystal form was dominated by kinetic effects in this region of the phase diagram.

Although a full study of the kinetics of crystallization is beyond the scope of this work, interesting information about the nature of crystal growth from the fluid can be obtained (a)

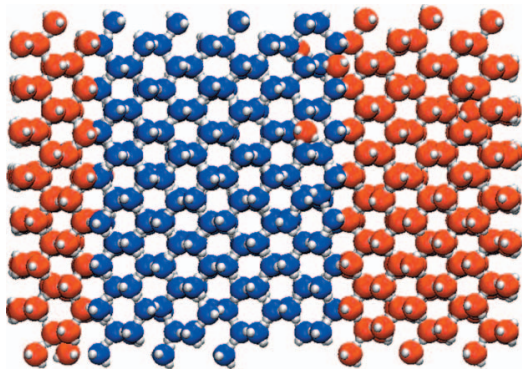

(b)

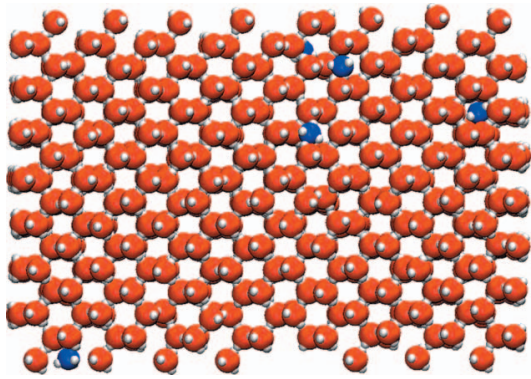

FIG. 9. Final configuration of the direct coexistence simulations of a fluiddiamond interface at $T^{*}=0.1$ and $p^{*}=0.05$ for the 12-6 model. Initially the simulation box contained 512 molecules in a diamond solid (i.e., $4 \times 4 \times 4$ unit cells) plus another 512 molecules in the fluid phase. Two different representations are shown. In (a) molecules that were in the diamond crystal in the initial configuration are colored in blue, whereas those that were fluid molecules in the initial configuration are shown in red. In (b) molecules that belong to the same diamond sublattice are colored in red, whereas those molecules not connected to the sublattice (i.e., defects) are colored in blue. As can be seen, the diamond crystal grows with a small number of defects.

from additional direct coexistence simulations. First, we examine whether a diamond crystal can continue to grow with this structure even though it is metastable with respect to the bcc solid. The interface was simulated for the 12-6 model at $T^{*}=0.1$ and $p^{*}=0.05$, a thermodynamic state at which the bcc solid is the most stable phase [see the red dot in Fig. 4(b)]. Figure 9 clearly shows that the crystal growth maintains the diamond lattice albeit with some defects. This result is in keeping with previous work, ${ }^{37}$ where it was shown that a diamond crystal could be grown by introducing a crystalline seed with diamond structure into the simulation box.

Second, we examine the nature of the crystal growth on a bec crystal under the same low pressure conditions. This was motivated by our hunch that the bcc/fluid interface might nucleate a diamond crystal that is coherent with the bcc lattice. Our reasoning was that if a defect or fluctuation leads to one of the diamond sublattices of the bcc crystal outgrowing the other, it may be hard to restore the bcc structure at the interface because diffusion of particles through the other sublattice would be very slow or even unfeasible. Instead, the "selected" sublattice would be more likely to continue to grow, leading to a diamond crystal. The results of the direct coexistence simulations of a bcc/fluid interface at low pressure reported in Fig. 10 confirm this scenario.

We checked that the growth of the diamond crystal from the bcc solid was not an artifact caused by the small size of the system by performing simulations for larger system sizes, which included both enlarging the area of the interface and the distance between the two interfaces in the simulation box. In all these examples the fluid in contact with the bcc 
(a)

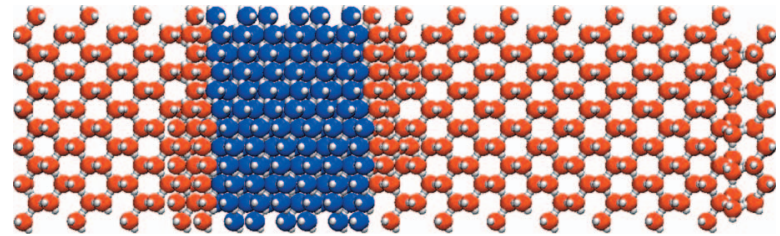

(b)

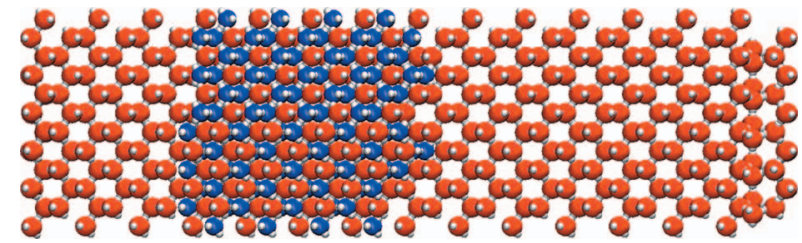

FIG. 10. Final configuration of the direct coexistence simulations of a fluidbcc interface at $T^{*}=0.1$ and $p^{*}=0.05$ for a simulation box containing 1296 molecules for the 12-6 model. Initially the simulation box contained 432 molecules in a bcc solid (i.e., $6 \times 6 \times 6$ unit cells) and 864 molecules of fluid. (a) and (b) show two different representations of the final configuration of the simulation, in which all the fluid has crystallized. (a) The molecules that were in the bcc solid structure in the initial configuration are colored in blue, whereas those that were fluid molecules in the starting configuration are colored in red. It can be seen that almost all the fluid has solidified into a diamond crystal. Only one or two incomplete bcc layers form at the two bcc-fluid interfaces. Most likely the defects in these first layers make less and less probable the growth of the bcc solid. (b) As mentioned in the manuscript a bcc solid is formed by two interpenetrating diamond solids. The two sublattices are highlighted by coloring the particles belonging to each sublattice in a different color, red for one sublattice and blue for the other. It can be seen that in this particular example, the same sublattice grew from each of the two interfaces. However, when the two diamond crystals growing from the two interfaces meet, some defects appear because as some particles were used to form one or two incomplete bcc layers at the bcc-fluid interfaces, the number of available particles is incommensurate with the dimensions of the simulation box (even though we chose it to be commensurate)

solid crystallized in a diamond crystal (the final configurations are depicted in the supplementary material ${ }^{60}$ ). In addition, we performed simulations for the shorter-ranged 20-10 $\mathrm{LJ}$ model at $T^{*}=0.10$ and $p^{*}=0.05$ (for which bcc is the most stable phase) and again it is observed that the bcc solid grows into a diamond crystal. The final configuration is provided as supplementary material. ${ }^{60}$

The growth of a bcc solid in contact with the fluid into a diamond crystal is a stochastic process and, therefore, depending on the initial conditions, the diamond crystal can grow following many possible different paths. Even though a detailed analysis of how the diamond crystal grows is beyond the scope of this work, some useful information can be obtained by inspecting the final configuration of our simulations. In all the simulations performed we observe that one or two incomplete bcc layers grew on the two faces of the bcc solid that were in contact with the fluid (snapshots are provided in the supplementary material ${ }^{60}$ ). As discussed before, the vacancies that are left on these two layers are most likely responsible for the growth of a diamond sublattice. It is observed that the diamond crystal grows from both interfaces. In the example shown in Fig. 10 the same diamond lattice grew from both interfaces. However, it is also possible that a different sublattice grows from each interface and an example is provided in the supplementary material. ${ }^{60}$ As there is no any reason why the same lattice should grow from the two sublattices, there is a $50 \%$ probability that they would be the same and $50 \%$ probability that they would be different. It is also observed that the amount of growth from each interface is often different and that when the crystals grown from the two interfaces meet usually some defects appear irrespective of whether the same or a different sublattice has grown from the two interfaces.

These results have important consequences for the formation of a diamond crystal from patchy tetrahedral particles, suggesting that the diamond phase may be able to form even when it is metastable with respect to other crystal structures. For example, even if the low pressure nucleation kinetics were to favor the formation of bcc nuclei, these might well then grow into diamond crystals. Furthermore, even if the low pressure nucleation kinetics were so slow that the system instead formed a glass (as perhaps suggested by our previous annealing simulations ${ }^{10}$ ), an alternative pathway might be to use a bcc crystal that was generated at higher pressure as a seed.

\section{CONCLUSIONS}

The phase diagram of model tetrahedral patchy particles was obtained from free energy calculations. Even though the width of the patches was narrow enough for the low pressure crystal form to be dominated by the energetics of specific patch-patch interactions, our results indicate that the diamond crystal is only thermodynamically stable when the range of the potential is below a critical value. At low pressures and finite temperatures, the diamond is competitive with a bcc solid, which consists of two interpenetrating diamond lattices. In a diamond lattice, there is enough empty space to interpenetrate another diamond lattice without repulsive energy between the two sublattices, thus obtaining a bcc crystal. Therefore, both the diamond and the bcc exhibit very similar energies, but the bcc is stabilized over the diamond lattice because of its lower enthalpy (i.e., lower value of the $p V$ term). Only at finite temperatures can the higher entropy of the diamond crystal make it more stable than the bcc solid. Our results show that the difference in entropy between the bcc and diamond solids increases as the range of the interactions decreases. As a consequence, the diamond solid is only stabilized when the range of the interactions is below some given value.

For the short-ranged model, the diamond solid is only stable at low pressures and finite temperatures. Upon compression the diamond transforms into the bcc solid. The rest of the phase diagram is qualitatively similar for the two ranges studied, although there are some quantitative differences. It is found that, upon compression, the bcc crystal transforms into an ordered fcc crystal, which exhibits a somewhat higher energy because in this case the four patches cannot be simultaneously perfectly aligned to four nearest neighbors. This transition moves to higher pressures as the range of the potential decreases. At high temperatures, the fluid freezes into a fcc plastic crystal.

The structure of our phase diagrams show strong similarities to those computed by Romano et al. for a similar tetrahedral patchy model. In particular, for both models the diamond structure is stabilized with respect to the bcc solid 
as the range of the potential is decreased. However, the differences between the two models - the region of stability for the diamond structure is smaller for our model-also highlight that as well as the symmetry of the particles, the particular "shape" of the interparticle potential can also have a strong effect on the phase behavior and on the stabilization of the diamond structure. What type of potential is likely to be representative of the patchy colloids that experimental groups are seeking to produce is not yet clear-it will depend on how the different surfaces of the patchy colloids are functionalized in order to generate selective attractions between the patches.

Even though we found that simple anisotropic models can stabilize the diamond solid, the diamond phase is only stable for a very narrow range of pressures. This behavior is in contrast with many water models for which ice Ic (diamond structure) is found to be more stable than ice VII (bcc structure) over a wider region of the phase diagram. ${ }^{69,70}$ These water models consist of a LJ at the oxygen site plus two positive point charges on the hydrogen sites and a negative charge whose location depends on the particular model. The stabilization of the diamond structure is related to the penetrability of the water model. The hydrogen bond distance is about $2.7 \AA$, whereas the $\mathrm{LJ} \sigma$ parameter is $3.15 \AA$. As a consequence, the interpenetration of a second diamond sublattice has a large energy penalty in water.

If one wants to nucleate a particular crystal from the fluid phase, both kinetic and thermodynamic effects must be considered. For the long-ranged 12-6 LJ model, we have found that a diamond crystal can be grown by introducing a crystalline seed (be it of diamond or bcc structure) into the simulation box. This is good news for those seeking to produce colloidal diamond using patchy colloids.

It would be interesting to perform nucleation studies on this system to further understand the crystallization behavior. As seen in the previous work, the nucleation of a diamond crystal is likely to be a challenging problem because, besides the bcc solid, the local structure in the liquid frustrates the nucleation of either the diamond or the bcc crystal. ${ }^{10}$ The phase diagram calculated in this work is a necessary precursor to such nucleation studies.

After submitting this manuscript, Romano et al. ${ }^{71}$ published an extended version of their previous work on the calculation of the phase diagram of tetrahedral particles described with the KF model. ${ }^{36}$ They also proposed an explanation for the higher vibrational entropy of the diamond crystal with respect to the bcc solid, which is in line with the discussion in the present manuscript.

\section{ACKNOWLEDGMENTS}

This work was funded by Grant No. FIS2007-66079C02-01 of Dirección General de Investigación Grant No. MODELICO-CM P2009/ESP-1691 of Comunidad Autónoma de Madrid, and Grant No. 2009801099 of CSIC. J.P.K.D. and A.A.L. are grateful for financial support from the Royal Society. C.V. thanks Francesco Sciortino and Flavio Romano for useful discussions during C.V.'s visit to the University of Rome and for sending him (Ref. 71) prior to publication. E.G.N. also acknowledges useful discussions with Noé G. Almarza.

${ }^{1}$ R. P. Sear, J. Chem. Phys. 111, 4800 (1999).

${ }^{2}$ X. Song, Phys. Rev. E 66, 011909 (2002).

${ }^{3}$ N. M. Dixit and C. F. Zukoski, J. Chem. Phys. 117, 8540 (2002).

${ }^{4}$ N. Kern and D. Frenkel, J. Chem. Phys. 118, 9882 (2003).

${ }^{5}$ J. Chang, A. M. Lenhoff, and S. I. Sandler, J. Chem. Phys. 120, 3003 (2004).

${ }^{6}$ V. Talanquer, J. Chem. Phys. 120, 084704 (2004).

${ }^{7}$ D. Rosenbaum, P. C. Zamora, and C. F. Zukoski, Phys. Rev. Lett. 21, 1407 (1996)

${ }^{8}$ P. R. ten Wolde and D. Frenkel, Science 277, 1975 (1997).

${ }^{9}$ B. W. Matthews, J. Mol. Biol. 33, 491 (1968).

${ }^{10}$ J. P. K. Doye, A. A. Louis, I.-C. Lin, L. R. Allen, E. G. Noya, A. W. Wilber, H. C. Kok, and R. Lyus, Phys. Chem. Chem. Phys. 9, 2197 (2007).

${ }^{11}$ H. Liu, S. K. Kumar, and F. Sciortino, J. Chem. Phys. 127, 084902 (2007).

${ }^{12}$ H. Liu, S. K. Kumar, F. Sciortino, and G. T. Evans, J. Chem. Phys. 130, 044902 (2009).

${ }^{13}$ J. J. McManus, A. Lomakin, O. Ogun, A. Pande, M. Basan, J. Pande, and G. B. Benedek, Proc. Natl. Acad. Sci. U.S.A. 104, 16856 (2007).

${ }^{14}$ C. Gögelein, G. Nägele, R. Tuinier, T. Gibaud, A. Stradner, and P. Schurtenberger, J. Chem. Phys. 129, 085102 (2008).

${ }^{15}$ X. Li, J. D. Gunton, and A. Chakrabarti, J. Chem. Phys. 131, 115101 (2009).

${ }^{16}$ O. Galkin and P. G. Vekilov, J. Am. Chem. Soc. 122, 156 (2000).

${ }^{17}$ H. R. Sheu, M. S. El-Aasser, and J. W. Vanderhoff, J. Polym. Sci. A 28, 629 (1990).

${ }^{18}$ V. N. Manoharan, M. T. Elsesser, and D. J. Pine, Science 301, 483 (2003).

${ }^{19}$ A. M. Jackson, J. W. Myerson, and F. Stellacci, Nature Mater. 3, 330 (2004).

${ }^{20}$ Y.-S. Cho, G.-R. Yi, J.-M. Li, S.-H. Kim, V. M. Manoharan, D. J. Pine, and S. M. Yang, J. Am. Chem. Soc. 127, 15968 (2005).

${ }^{21}$ Y.-S. Cho, G.-R. Yi, S.-H. Kim, D. J. Pine, and S. M. Yang, Chem. Mater. 17, 5006 (2005).

${ }^{22}$ K.-H. Roh, D. C. Martin, and J. Lahann, Nature Mater. 4, 759 (2005).

${ }^{23}$ C. E. Snyder, A. M. Yake, J. D. Feick, and D. Velegol, Langmuir 21, 4813 (2005)

${ }^{24}$ Z. F. Li, D. Y. Lee, M. F. Rubner, and R. E. Cohen, Macromolecules 38, $7876(2005)$.

${ }^{25}$ A. van Blaaderen, Science 439, 545 (2006).

${ }^{26}$ K.-H. Roh, D. C. Martin, and J. Lahann, J. Am. Chem. Soc. 128, 6796 (2006).

${ }^{27}$ G. A. DeVries, M. Brunnbauer, Y. Hu, A. M. Jackson, B. Long, B. T. Neltner, O. Uzun, B. H. Wunsch, and F. Stellacci, Science 315, 358 (2007).

${ }^{28}$ Y.-S. Cho, G.-R. Yi, S.-H. Kim, S.-J. Jeon, M. T. Elsesser, H. K. Yu, S.-M. Yang, and D. J. Pine, Chem. Mater. 19, 3183 (2007).

${ }^{29}$ L. Hong, A. Cacciuto, E. Luijten, and S. Granick, Langmuir 24, 621 (2008).

${ }^{30}$ A. Perro, E. Duguet, O. Lambert, J.-C. Taveau, E. Bourgeat-Lami, and S. Ravaine, Angew. Chem., Int. Ed. 121, 367 (2009).

${ }^{31}$ D. J. Kraft, J. Groenewold, and W. K. Kegel, Soft Matter 5, 3823 (2009).

${ }^{32}$ S. C. Glotzer and M. J. Solomon, Nature Mater. 6, 557 (2007).

${ }^{33}$ S. Yang, S.-H. Kim, J.-M. Lim, and G.-R. Yi, J. Mater. Chem. 18, 2177 (2008).

${ }^{34}$ A. B. Pawar and I. Kretzschmar, Macromol. Rapid Commun. 31, 150 (2010).

${ }^{35}$ E. G. Noya, C. Vega, J. P. K. Doye, and A. A. Louis, J. Chem. Phys. 127, 054501 (2007).

${ }^{36}$ F. Romano, E. Sanz, and F. Sciortino, J. Phys. Chem. B 113, 15133 (2009).

${ }^{37}$ Z. Zhang, A. S. Keys, T. Chen, and S. C. Glotzer, Langmuir 21, 11547 (2005).

${ }^{38}$ G. Doppelbauer, E. Bianchi, and G. Kahl, J. Phys.: Condens. Matter 22, 104105 (2010).

${ }^{39}$ M. F. Hagan and D. Chandler, Biophys. J. 91, 42 (2006).

${ }^{40}$ A. W. Wilber, J. P. K. Doye, A. A. Louis, E. G. Noya, M. A. Miller, and P. Wong, J. Chem. Phys. 127, 085106 (2007)

${ }^{41}$ A. W. Wilber, J. P. K. Doye, and A. A. Louis, J. Chem. Phys. 131, 175101 (2009). 
${ }^{42}$ A. W. Wilber, J. P. K. Doye, A. A. Louis, and A. C. F. Lewis, J. Chem. Phys. 131, 175102 (2009).

${ }^{43}$ A. Giacometti, F. Lado, J. Largo, G. Pastore, and F. Sciortino, J. Chem. Phys. 131, 174114 (2009).

${ }^{44}$ F. Sciortino, A. Giacometti, and G. Pastore, Phys. Rev. Lett. 103, 237801 (2009).

${ }^{45}$ M. Maldovan, C. K. Ullal, W. C. Carter, and E. L. Thomas, Nature Mater. 2, 664 (2003)

${ }^{46}$ M. Maldovan and E. L. Thomas, Nature Mater. 3, 593 (2004).

${ }^{47}$ A.-P. Hynninen, J. H. J. Thijssen, E. C. M. Vermolen, M. Dijkstra, and A. van Blaaderen, Nature Mater. 6, 202 (2007).

${ }^{48}$ C. Vega, E. Sanz, J. L. F. Abascal, and E. G. Noya, J. Phys.: Condens. Matter 20, 153101 (2008).

${ }^{49}$ C. Vega and E. G. Noya, J. Chem. Phys. 127, 154113 (2007).

${ }^{50}$ E. G. Noya, M. M. Conde, and C. Vega, J. Chem. Phys. 129, 104704 (2008).

${ }^{51}$ D. Frenkel and B. Smit, Understanding Molecular Simulation: From Algorithms to Applications (Academic, Boston, 1996).

${ }^{52}$ D. Frenkel and A. J. C. Ladd, J. Chem. Phys. 81, 3188 (1984).

${ }^{53}$ J. M. Polson, E. Trizac, S. Pronk, and D. Frenkel, J. Chem. Phys. 112, 5339 (2000)

${ }^{54}$ J. W. Schroer and P. A. Monson, J. Chem. Phys. 112, 8950 (2000).

${ }^{55}$ D. A. Kofke, J. Chem. Phys. 98, 4149 (1993).

${ }^{56}$ D. A. Kofke, Mol. Phys. 78, 1331 (1993).

${ }^{57}$ W. Vetterling, S. Teukolsky, W. Press, and B. Flannery, Numerical Reci- pes. Example Book (Fortran) (Cambridge University Press, Cambridge, 1985).

${ }^{58}$ A. J. C. Ladd and L. V. Woodcock, Chem. Phys. Lett. 51, 155 (1977).

${ }^{59}$ A. J. C. Ladd and L. V. Woodcock, Mol. Phys. 36, 611 (1978).

${ }^{60}$ See supplementary material at http://dx.doi.org/10.1063/1.3454907 for tables of free energies and coexistence points and for snapshots of the final configurations of direct coexistence simulations.

${ }^{61}$ J. Kolafa and I. Nezbeda, Mol. Phys. 61, 161 (1987).

${ }^{62}$ C. Vega and P. A. Monson, J. Chem. Phys. 109, 9938 (1998).

${ }^{63}$ F. Romano, P. Tartaglia, and F. Sciortino, J. Phys.: Condens. Matter 19, 322101 (2007).

${ }^{64}$ J. L. Aragones, E. G. Noya, J. L. F. Abascal, and C. Vega, J. Chem. Phys. 127, 154518 (2007).

${ }^{65}$ E. Whalley, J. Chem. Phys. 81, 4087 (1984).

${ }^{66}$ W. Ostwald, Z. Phys. Chem. 22, 289 (1897)

${ }^{67}$ I. Stranski and D. Totomanow, Z. Phys. Chem. 163, 399 (1933).

${ }^{68}$ P. R. ten Wolde and D. Frenkel, Phys. Chem. Chem. Phys. 1, 2191 (1999).

${ }^{69}$ E. Sanz, C. Vega, J. L. F. Abascal, and L. G. MacDowell, Phys. Rev. Lett. 92, 255701 (2004).

${ }^{70}$ C. Vega, J. L. F. Abascal, E. Sanz, L. G. MacDowell, and C. McBride, J. Phys.: Condens. Matter 17, S3283 (2005).

${ }^{71}$ F. Romano, E. Sanz, and F. Sciortino, J. Chem. Phys. 132, 184501 (2010). 